\title{
On the facultative requirement of the bacterial RNA chaperone, Hfq
}

\section{Ambre Jousselin, Laurent Metzinger and Brice Felden}

Université de Rennes I, Inserm U835, UPRES EA2311, Biochimie Pharmaceutique, 2 avenue du Prof. Léon Bernard 35043 Rennes, France

The pleiotropic post-transcriptional regulator $\mathrm{Hfq}$ is an RNA chaperone that facilitates pairing interactions between small regulatory RNAs (sRNAs) and their mRNA targets in several bacteria. However, this classical pattern, derived from the Escherichia coli model, is not applicable to the whole bacterial kingdom. In this article we discuss the facultative requirement for $\mathrm{Hfq}$ for SRNAmRNA duplex formation among bacteria and the specific features of the Hfq protein and RNA duplexes that might account for the dispensability or requirement of the chaperone. Apparent links between the need for $\mathrm{Hfq}$, the GC content of bacterial genomes and the free energy of experimentally validated sRNA-mRNA pairing interactions are presented.

Is Hfq involved in all sRNA-mRNA interactions?

Bacterial small ribonucleic acids (sRNAs) that are not ribosomal, transfer or messenger RNAs play central roles in the regulation of gene expression in response to environmental changes [1]. Some sRNAs are also implicated in bacterial virulence [2]. The majority of the known sRNAs interact near, or at, the translation start site of their dedicated mRNA targets to exert mostly negative effects by RNA silencing, inducing mRNA decay and/or translation inhibition. Overlapping networks of interactions are common and experimentally validated pairings are usually found to be imperfect and to contain mismatches and unpaired nucleotides. In some bacterial species, such interrupted pairings are assisted by a dedicated RNA chaperone known as Hfq (host factor required for phage $\mathrm{Q} \beta$ RNA replication) that belongs to the $\mathrm{Sm}$ family of proteins [3-5].

Approximately half of the sequenced bacterial genomes encode a Hfq homolog [4]. Hfq proteins contain an Nterminal $\alpha$-helical domain followed by an antiparallel five-stranded $\beta$-sheet and form stable hexameric ringshaped structures of identical subunits [5]. They preferentially bind to A/U-rich single strands close to hairpins in RNAs [6]. Hfa is one of the most abundant proteins in Escherichia coli (30 000-60 000 per cell [7]) where it modulates the stability, translation and polyadenylation of many mRNAs [8], as well as RNA processing events. Defects including reduced growth, impaired resistance to various stresses and altered virulence are detected in $E$. coli cells lacking Hfa [9]. However, in some bacteria, such as Staphylococcus aureus, $h f q$ is transcribed at very low levels during growth [10], although sRNA-mRNA interactions are known to regulate the expression of several genes in this microorganism [11].

In this article we discuss the role of the bacterial Hfq protein in RNA-mediated gene regulation and highlight its dispensability in numerous cases. For our purpose, only experimentally supported sRNA-mRNA pairings (evidenced by structural probing and/or extended mutational analysis) are provided as examples. Structural variations in Hfq proteins throughout phylogeny are also considered. Here we propose that the need for Hfq is linked to the GC content of the bacterial genomes and the free energy of experimentally supported sRNA-mRNA pairing interactions.

Functions of the Hfq protein in sRNA-mediated gene regulation

$E$. coli is a paradigm for the importance of Hfq in sRNAbased gene regulation. At least $22 E$. coli sRNAs require Hfa to function [12], including all trans-acting antisense sRNAs with the exceptions of the IstR-1/tisB [13], IpeX/ ompC [14] and SymR/symE RNA hybrids [15] (by convention, hybrids are named in the form sRNA $/ m R N A$ ). The sRNAs base pair with their target mRNAs and modulate gene expression by modifying mRNA decay and/or translation initiation. In $E$. coli, Hfq induces structural changes in the target mRNA and/or the sRNA. A study using realtime fluorescence resonance energy transfer (FRET) measurements on the interaction between truncated versions of the DsrA sRNA and the rpoS mRNA showed that Hfq accelerates strand exchange and subsequent annealing between the sRNA and its target mRNA [16]. The protein strongly enhances the rate of sRNA-mRNA duplex formation, enabling the sRNA to compete efficiently with ribosome binding.

\footnotetext{
Glossary

Antisense RNA: an RNA that interacts with a complementary mRNA target to modify its expression.

Degradosome: a multi-protein complex involved in the degradation of various RNAs.

Riboregulation: regulation exerted by an RNA.

RNA silencing: downregulation of gene expression by an RNA molecule. RNome: all the nonconventional RNAs (mRNAs, tRNAs and rRNAs excluded) expressed by an organism.

Sm family of proteins: RNA-binding proteins that are involved in mRNA splicing in eukaryotes and archaea.

Trans-acting RNAs: RNAs that bind target mRNA sequences via imperfect pairings to regulate their expression levels.
} 
In Salmonella enterica serovar Typhimurium, highthroughput pyrosequencing technology has recently identified several sRNAs that are enriched by co-immunoprecipitation with Hfq [17]. In an independent study, Viegas and collaborators showed that a Hfq mutation reduces the half-life of two sRNAs expressed in this bacterium, SraL and MicA [18]. Interestingly, these sRNAs are conserved in several enterobacteria. The steady-state levels of two Pseudomonas aeruginosa $\mathrm{sRNAs}, \mathrm{PhrD}$ and $\mathrm{PhrS}$, are also both decreased by half in a $h f q$ mutant, whereas their half-life remains unchanged [19]. This suggests that Hfq affects their transcription levels, although this might be an indirect effect. It should be noted that a lower level of a particular sRNA in a $h f q$ mutant does not necessarily imply a role for Hfq in the formation of the corresponding sRNA-mRNA hybrid. In $E$. coli, however, most of the sRNAs requiring Hfq for their functions have a shorter half-life in $h f q$ deletion strains [20]. In several gammaproteobacteria, Hfq is required for numerous sRNA-based gene regulations (Figure 1a) [1].

In E. coli, there is a functional link between $\mathrm{Hfq}$ and the single-strand specific endoribonuclease E (RNase E). Gene silencing by sRNAs is triggered by a ribonucleoprotein complex containing RNase $\mathrm{E}$ that interacts with Hfq to cause rapid target mRNA degradation and translation repression [21]. Hfq interacts with the C-terminal domain of RNase $\mathrm{E}$ and the RNase cleaves the target mRNAs [21] to initiate their rapid decay. The Hfq binding sites on sRNAs coincide with the RNase E cleavage sites [22]. Therefore, Hfq might protect sRNAs from endonucleolytic attack, which would explain why Hfq increases the half-life of most sRNAs with which it interacts [20]. The protein also stabilizes Hfq-interacting sRNAs in bacteria other than $E$. coli, such as Listeria monocytogenes [23] and $P$. aeruginosa [24]. In cases for which Hfq is involved in sRNA-mRNA interactions, it can facilitate recruitment of RNase $\mathrm{E}$ from the degradosome for mRNA decay (exceptions exist, such as the $E$. coli sRNA Spot42, which does not induce degradation of its target mRNA galK [20]). In $E$. coli, degradation of repressed mRNAs is probably a consequence of ribosome exclusion rather than the primary event because translational repression can occur without mRNA destabilization [25], implying that RNAse E might be dispensable for translation silencing. There is an interesting parallel with the situation in $S$. aureus: regulatory RNAIII (an sRNA) causes direct translation repression of target mRNAs and induces their degradation in a process dependent on the double-strand specific endoribonuclease III (RNase III) [11]. RNAIII-dependent inhibition of ribosome loading onto the target mRNAs is coupled to RNase

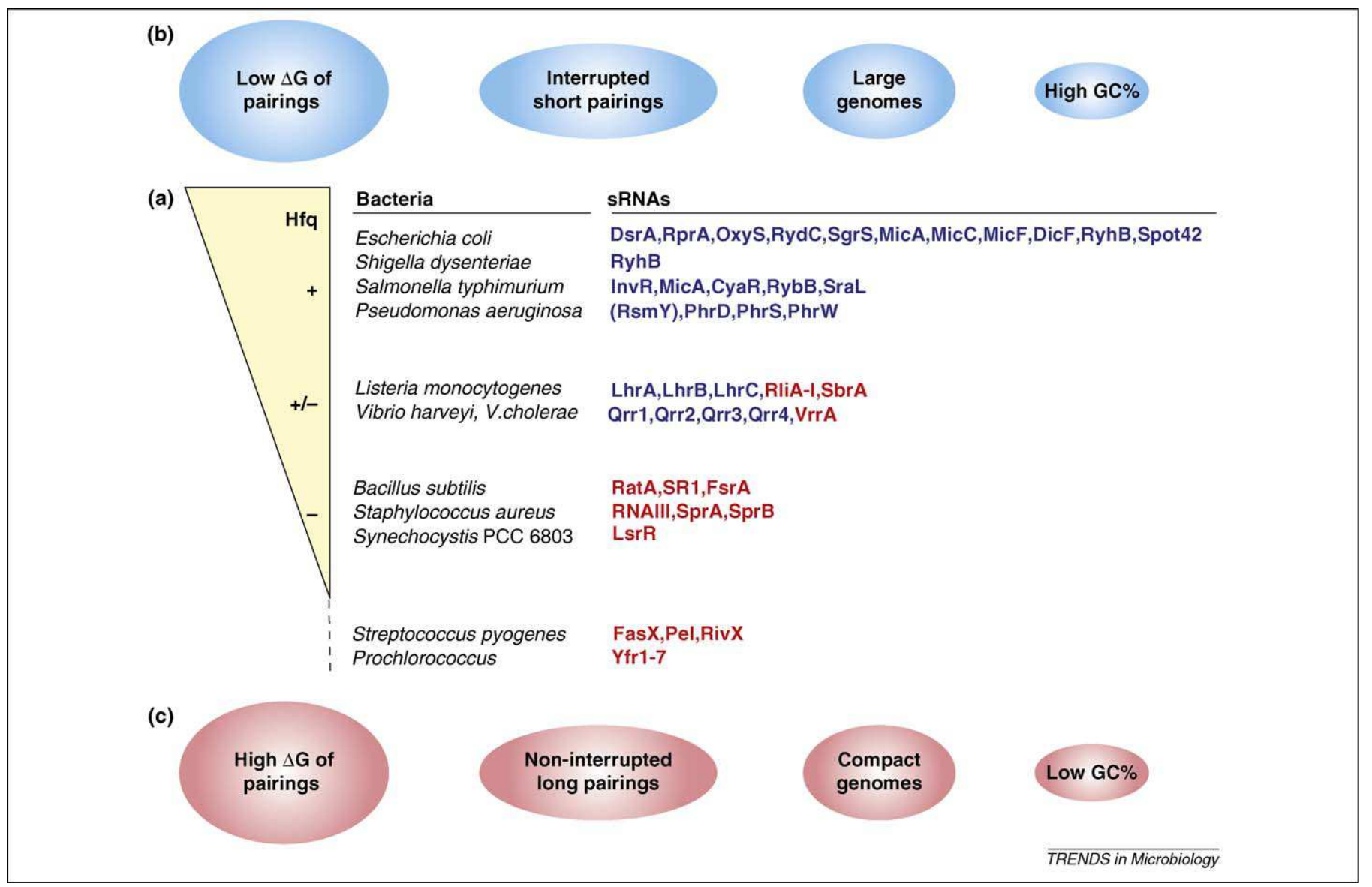

Figure 1. Variable requirement for Hfq in sRNA-mediated gene regulations in bacteria. (a) List of bacteria and sRNAs organized according to their Hfq requirement. With the

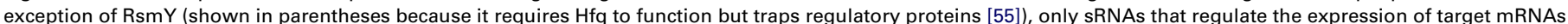

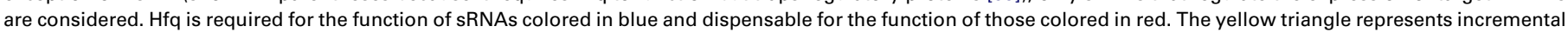
involvement of Hfq. The hfq gene is missing in S. pyogenes and in most strains of Prochlorococcus. Note that in E. coli [56] and S. typhimurium [17] additional sRNAs interact with $\mathrm{Hfq}$ in vivo. (b,c) Parameters to consider for the facultative involvement of the Hfq protein in sRNA-mRNA interactions. The size of the circles reflects the relative importance of each parameter. 
III-dependent cleavage, probably to render translation arrest irreversible.

\section{In the same bacterium, Hfq can be required for some sRNAs and dispensable for others}

In the human pathogen Vibrio cholerae and the marine bacterium Vibrio harveyi, Hfq creates a regulatory switch with four redundant sRNAs (Qrr1-Qrr4) impacting gene expression at high cellular density [26]. At least some of the Qrr RNAs in V. cholerae act by base pairing on multiple target mRNAs and require Hfq for function [27]. Another sRNA from $V$. cholerae, VrrA, downregulates ompA porin mRNA by base pairing with the $5^{\prime}$ region of the mRNA [28]. However, VrrA-mediated regulation of OmpA expression occurs in the absence of Hfq in vivo, indicating that the protein is dispensable for the action of VrrA [28].

This non-uniform action of Hfq also seems to be true for $L$. monocytogenes. In this Gram-positive food-borne pathogen, three sRNAs (LhrA-LhrC) co-immunoprecipitate with Hfq [23], suggesting that the protein participates in their functions. However, RliB, RliE and RliI sRNAs from the same bacterium are able to interact with their mRNA targets in vitro without Hfo [29]. It should be noted, however, that a possible in vivo role for Hfq cannot be ruled out for these sRNAs based only on in vitro studies. The functions of these sRNAs are unclear and minor effects on expression levels of their putative target mRNAs were detected after sRNA overexpression, suggesting indirect regulation. Another recently identified SRNA, SbrA, which is part of the $\sigma^{\mathrm{B}}$ regulon (involved in response to stress) of $L$. monocytogenes, does not interact with Hfq and most probably functions independently of this RNA chaperone [30]. The above examples from Vibrio and Listeria, as well as the cases from E. coli (IstR, IpeX and SymE) already mentioned, illustrate how non-uniform the involvement of Hfq can be in sRNAmRNA mediated regulations within the same bacterium.

\section{Hfq as a dispensable factor in sRNA-controlled gene regulations}

In the low-GC Gram-positive S. aureus, the $h f q$ gene is present but expressed at a very low level during growth [10], which contrasts with the abundance of the Hfa protein in $E$. coli. However, $S$. aureus expresses numerous sRNAs $[31,32]$ with at least one, RNAIII, forming antisense pairings with several mRNAs [11]. The involvement of Hfq in RNAIII-mediated gene regulation was addressed experimentally but remains unclear. According to co-immunoprecipitation and band shift experiments, RNAIII binds specifically to Hfq in vivo and in vitro [33]. However, Hfq does not facilitate complex formation between RNAIII and one of its target mRNAs (spa) and $h f q$ deletion does not affect the in vivo level of spa mRNA or that of RNAIII [10]. Concerning additional mRNAs targets of RNAIII, it has been shown that Hfa is not involved in translational repression of rot mRNA [34] or in duplex formation between sa1000 mRNA and RNAIII [11]. This raises the question of the role of Hfq in the stability and function of other sRNAs in $S$. aureus.

Additional sRNAs are expressed by $S$. aureus [32], some of which (SprA and SprB) seem to regulate in vivo the expression of mRNAs by antisense pairing (Shabelskaya and Felden, unpublished observations). As assayed by gel retardation, complexes between either SprA or SprB transcripts and purified $S$. aureus Hfa seem to form, but they might be non-specific and they can be destabilized by addition of an excess of non-relevant RNAs such as tRNAs (Jousselin et al., unpublished observations). In addition, Northern blotting experiments revealed that Hfq does not seem to influence in vivo levels of SprA and SprB during both exponential and stationary growth phases (Jousselin et al., unpublished observations). Moreover, SprA interacts in vitro with its predicted SA2216 mRNA target in the absence of Hfq [32]. Finally, after testing more than 1000 different growth conditions, no detectable phenotypic difference was observed between the $h f q$ deletion mutant and wild type $S$. aureus [10]. All these data suggest that Hfq is dispensable for the known cases of sRNA-controlled gene regulation in $S$. aureus.

The chromosome of Bacillus subtilis encodes a Hfq-like RNA chaperone, YmaH [35], and several sRNAs under sporulation control [36]. SR1 is an sRNA involved in regulation of arginine catabolism by preventing translation of the $a h r C$ mRNA, encoding a transcriptional activator. The YmaH protein is dispensable for complex formation between SR1 and $a h r C$ mRNA in vitro and it does not influence the stability of SR1 in vivo, confirming its dispensability [37]. Structural probes revealed that the 3 -terminal stem-loop of SR1 unfolds to interact with $a h r C$ mRNA [38]. FsrA, another sRNA expressed by B. subtilis, represses expression of mRNAs encoding iron-containing enzymes such as succinate dehydrogenase $(s d h C)$ by pairing with $s d h C$ mRNA [39]. Its function does not require a contribution from YmaH. Therefore, the Hfq homolog in $B$. subtilis is not involved in the in vivo stability of known sRNAs. An as yet uncharacterized factor is possibly required to facilitate the interaction in vivo.

A Hfa homolog was identified in Synechocystis sp. PCC 6803 and there is also an antisense RNA (IsrR) that negatively controls expression of the accessory photosynthesis protein IsiA [40]. Preliminary results argue against a role of the Hfa homolog in riboregulation of photosynthesis because IsiA and IsrR transcript levels are not altered in a $\Delta h f q$ mutant. However, Hfq plays critical roles in the expression of motility slr genes in Synechocystis [41]. These specific examples indicate that Hfq, or Hfq homologs, can be dispensable for sRNA stability in vivo. Again, this contrasts with the situation in Gram-negative E. coli, in which Hfq influences the stability of most of the transacting sRNAs in vivo.

\section{Rationalizing the facultative requirement for $\mathrm{Hfq}$ in bacterial sRNA-mRNA interactions}

Genomic GC content

The implication of Hfq in sRNA-mRNA interactions varies among bacteria and seems to be somewhat connected to the overall GC content of the genomic DNA (Table 1). It is striking that most known trans-acting antisense sRNAs in E. coli (with a $50 \%$ overall GC content) need Hfa for function [12], as is the case for the other known sRNAs in gammaproteobacteria (50-67\% overall GC content) $[17,19]$. However, for some bacteria with a GC content of $\sim 50 \%$, such as Vibrio cholerae (47\%), the involvement of 
Table 1. Relations between genome size, overall GC content and involvement of Hfq in RNA-controlled gene regulations in several bacteria

\begin{tabular}{|c|c|c|c|c|}
\hline Bacterial species & Genome size (Mb) & GC (\%) & Phylum & References \\
\hline Pseudomonas aeruginosa & 6 & 66 & Proteobacteria $(\gamma)$ & [19] \\
\hline Neisseria meningitidis & 2.2 & 51 & Proteobacteria $(\beta)$ & [58] \\
\hline Salmonella typhimurium & 4.8 & 45 & Proteobacteria $(\gamma)$ & {$[17,59,60]$} \\
\hline \multicolumn{5}{|c|}{ Hfq involved in the function of some sRNAs } \\
\hline \multicolumn{5}{|c|}{$h f q$ gene present but seems to be dispensable } \\
\hline Bacillus subtilis & 4.2 & 43 & Firmicutes & {$[38,39]$} \\
\hline Staphylococcus aureus & 2.8 & 32 & Firmicutes & {$[11,33]$} \\
\hline Synechocistis PCC6803 & 3 & 47.7 & Cyanobacteria & [41] \\
\hline Prochlorococcus marinus & 1.6 & 31 & Cyanobacteria & {$[49,50]$} \\
\hline \multicolumn{5}{|l|}{$h f q$ gene absent } \\
\hline Enterrococcus faecalis & 3.2 & 37 & Firmicutes & [1] \\
\hline Borrelia burgdorferri & 0.9 & 26 & Spirochaetes & [51] \\
\hline
\end{tabular}

${ }^{a}$ In E. coli, most sRNAs require Hfq for their functions, although three exceptions are known (see the text).

${ }^{\mathrm{b}}$ The genome of S. coelicolor, with a high GC content, lacks $h f q$ although it encodes several sRNAs; however, the functions of these sRNAs are unknown and might not depend on mRNA-sRNA pairing interactions.

Hfq is variable. Nevertheless, when considering bacteria with low-GC values, such as S. aureus ( $32 \%$ GC), Hfq seems to be dispensable. It seems that most bacteria requiring Hfq for some of their sRNA-controlled gene regulation possess an overall GC content $\geq 50 \%$ (Table 1). A possible explanation for this observation is that a higher GC content in interacting sRNAs and mRNAs is expected to result in more stable conformations, which would need to be loosened by a chaperone for a productive interaction to occur. We therefore hypothesize that low-GC bacteria usually do not need Hfq to enhance sRNA-mRNA duplex formation for efficient gene regulation (Figure 1b,c).

\section{Extended pairing might overcome the requirement for Hfq}

In $E$. coli, Hfq-dependent sRNA-mRNA duplexes are formed between relatively short RNA sequences interrupted by mismatches $[42,43]$. For instance, the experimentally supported RyhB/sodB and OxyS/fhlA interactions only involve 9 and $7 \mathrm{bp}$, respectively (Table 2). In these cases, Hfq enhances the rate of duplex formation between the interacting RNAs.

By contrast, some sRNA-mRNA hybrids form in the absence of the Hfq protein in vivo and in vitro, as is the case for several mRNA targets of RNAIII in $S$. aureus [11]. These sRNA-mRNA duplexes are usually longer than those requiring Hfq in $E$. coli. It seems that long duplexes might have sufficient stability, with RNA chaperones being dispensable. As examples, the interactions (experimentally supported by structural probing) between rot, spa and sa 1000 mRNAs and RNAIII involve 33, 44 and $50 \mathrm{bp}$, respectively [11]. These extended pairings seem to overcome the requirement for Hfq, as is also the case for the long and uninterrupted sRNA-mRNA hybrids in $E$. coli plasmids and antisense sRNAs [44].
Table 2 provides examples of sRNA-mRNA interactions in various bacteria that are experimentally supported by either structural probing and/or mutagenesis. In each case, we calculated the free energy of the interaction using the RNAup software, which considers the energy necessary to open the binding site and the energy gained from hybridization [45]. Remarkably, we found that pairing interactions with the lowest $\Delta G$ values (RyhB/sodB, GcvB/ $d p p A, \mathrm{OxyS} / f h l A, \mathrm{GcvB} / \mathrm{opp} A)$ need the contribution of Hfq for their regulation, whereas the Hfq protein is dispensable for those with the highest $\Delta G$ values (RNAIII/ sa1000 and IstR-1/tisAB). Therefore, we propose that the role of Hfq in bacterial sRNA-mRNA interactions depends, at least in part, on the free energy for pairings (Figure 1b,c). However, the $\Delta G$ values calculated should be confirmed experimentally by melting temperature measurements for each sRNA-mRNA duplex.

\section{Importance of the Hfq structure}

Structural variations among Hfq proteins throughout bacterial phylogeny might also account for their different contributions in sRNA-mRNA interactions. Hfq proteins display a conserved common core but considerable variation at their C-terminal ends, with the gammaproteobacteria possessing the longest C-terminal extensions [4]. Interestingly, it has been shown that a C-terminal extension of $E$. coli Hfq constitutes an mRNA interaction surface because a C-terminal truncated Hfq variant binds sRNAs but not mRNAs [46]. The Hfq proteins from $S$. aureus and B. subtilis have very short C-termini, so it could be speculated that they are unable to interact efficiently with the mRNA targets of the sRNAs, which might contribute to their dispensability for sRNA-mRNA interactions in these organisms. Nevertheless, the smallest known Hfq, which occurs in the archeon Methanocaldococcus jannaschii, 
Table 2. Relation between the free energy $(\Delta G)$ calculated for sRNA-mRNA pairings [63] and implication of Hfq in RNA-controlled gene regulations for RNA duplexes validated experimentally by structural probing and/or mutagenesis

\begin{tabular}{|c|c|c|c|c|c|}
\hline sRNA-mRNA & 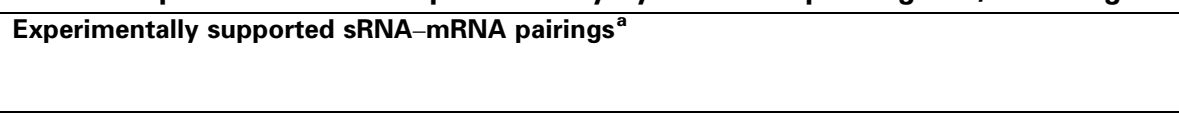 & $\begin{array}{l}\text { Pair } \\
\text { length } \\
\text { (bp) }\end{array}$ & $\begin{array}{l}\text { GC } \\
(\%)\end{array}$ & $\begin{array}{l}\Delta G \\
\text { (kcal/ } \\
\text { mol) }\end{array}$ & Refs \\
\hline \multicolumn{6}{|l|}{ Hfq required } \\
\hline RyhB/sodB ${ }^{\mathrm{b}}$ & 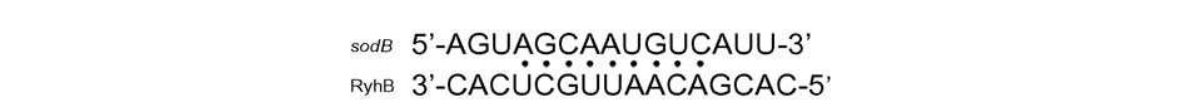 & 9 & 44 & -5.5 & [42] \\
\hline \multicolumn{6}{|c|}{ 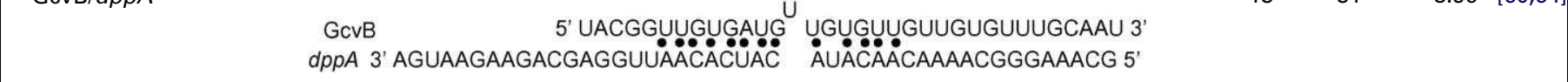 } \\
\hline OxyS/fhIA ${ }^{\mathrm{b}}$ & 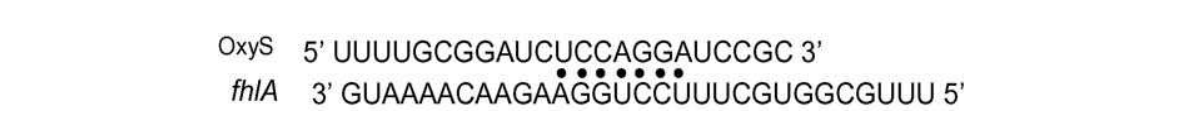 & 7 & 57 & -10.5 & {$[65]$} \\
\hline $\mathrm{GcvB} / o p p A^{\mathrm{b}}$ & UGU & 17 & 23 & -11.2 & {$[60,64]$} \\
\hline & 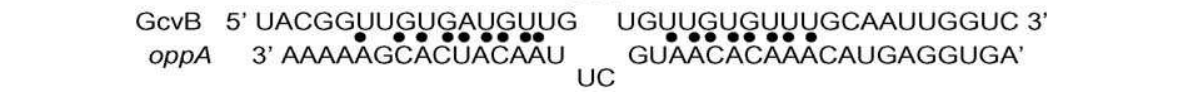 & & & & \\
\hline $\mathrm{PtsG} / \operatorname{sgrS}^{\mathrm{b}}$ & 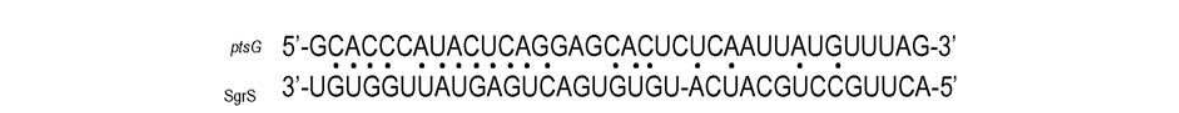 & 23 & 39 & -12.2 & [43] \\
\hline Spot42/galK ${ }^{b}$ & 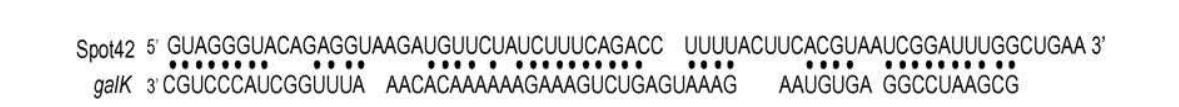 & 40 & $35-50$ & -16 & {$[66]$} \\
\hline $\mathrm{OmpA} / \mathrm{mic}^{\mathrm{b}}$ & $\begin{array}{l}\text { мmpA 5'-GAUGAUAACGAGGCGC-3' } \\
\text { MicA 3'-CUACUAUUGUUUCGCG-5' }\end{array}$ & 16 & 43 & -20 & [67] \\
\hline \multicolumn{6}{|l|}{ Hfq not required } \\
\hline $\mathrm{FsrA} / s d h C^{\mathrm{c}}$ & 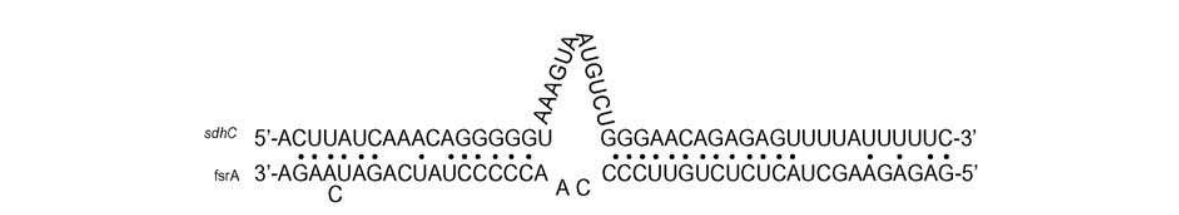 & 42 & 20 & -18 & [39] \\
\hline RNAIII/spa ${ }^{\mathrm{d}}$ & 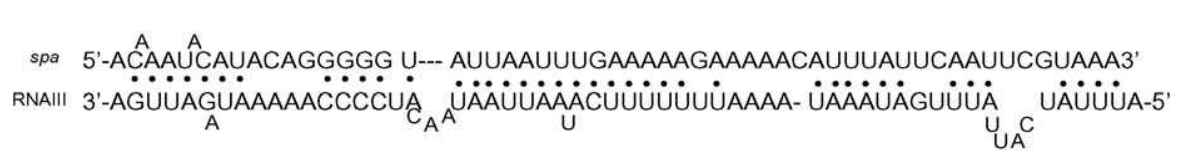 & 44 & 15.9 & -22.5 & [33] \\
\hline RNAIII/sa1000 d,e & 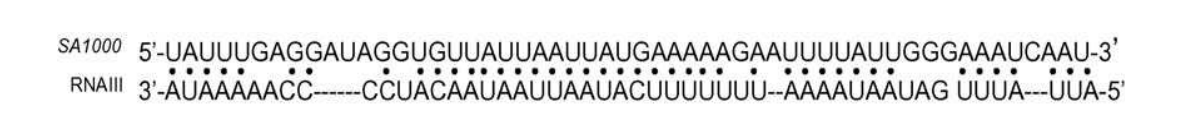 & 50 & 10 & -34.5 & {$[11]$} \\
\hline IstR-1/tis $A B^{\mathrm{b}}$ & 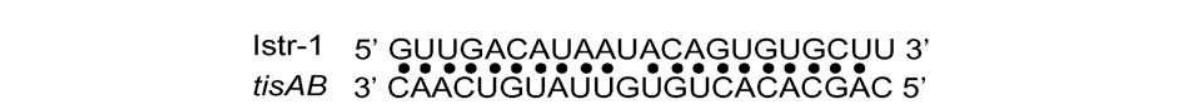 & 21 & 39 & -35 & [13] \\
\hline
\end{tabular}

${ }^{a}$ SR1/ahrC and rot/RNAll were not included in the table because the free energy for these two pairing interactions could not be calculated. SR1/ahrC relies on several short experimentally supported interrupted nucleotide stretches, some located at long distances from one another [38]. In addition, the experimentally supported loop-loop interactions between rot mRNA and RNAlll occur between distant portions of each of the two RNA partners [11].

${ }^{\mathrm{b}}$ From E. coli.

${ }^{\mathrm{c} F r o m ~ B . ~ s u b t i l i s . ~}$

${ }^{\mathrm{d}}$ From S. aureus.

${ }^{\mathrm{e}} \mathrm{sa} 1000$ is a fibrinogen-binding mRNA.

binds efficiently to $E$. coli sRNAs and possesses the ability to promote riboregulation via intermolecular base pairing and can complement, at least in part, a number of phenotypes displayed by an $E$. coli $h f q$ knockout strain [47].

Another important structural difference between $E$. coli and S. aureus Hfq proteins, which most likely impacts their functions, concerns their electrostatic potential energy surfaces and their proposed RNA binding sites. For E. coli Hfa, the positive electrostatic surface of the trough that connects the proximal and distal faces is in sharp contrast to the same area on $S$. aureus Hfa, which shows a negative electrostatic surface [48]. Variability in the Hfq core barrel could also account for non-conservation of the RNA chaperone function.

\section{Bypassing Hfq for sRNA functions}

In bacteria expressing an RNome, keeping a functional Hfq chaperone is probably an advantage to enable a large set of sRNA-mRNA interactions to occur, including those that are energetically unfavorable. It is remarkable that some 
bacteria, such as $S$. aureus, possess a copy of the $h f q$ gene but the encoded protein does not seem to play any significant role in the cell; this is in agreement with the very low transcription levels, as discussed in a previous section. It is possible that some unidentified mutations in the promoter of the $h f q$ gene might be responsible for the weak transcription yields. Alternatively, $h f q$ could be expressed at high levels under restricted, and currently unknown, growth conditions.

The $h f q$ gene could be in the process of being erased in certain bacterial lineages. In Prochlorococcus, $h f q$ is present in only two out of 12 sequenced strains [49]. Nevertheless, the naturally $h f q$-deficient Prochlorococcus MED4 expresses at least 24 sRNAs [50]. The absence of $h f q$ in MED4 suggests that either the antisense sRNAs detected function without chaperone support or that a novel nonHfq chaperone is yet to be identified.

Despite the absence of $h f q$ in its genome, the etiological agent of Lyme disease, Borrelia burgdorferi, contains several sRNAs [51]. Hfq homologs are also absent in several low-GC Gram-positive bacteria such as Streptococcus pyogenes, which expresses at least three sRNAs: FasX [52], RivX [53] and Pel [54]. All three sRNAs regulate the expression of virulence factors. The mechanism of action of these sRNAs is unknown and their functions might not require the help of a chaperone. Some bacteria lacking $h f q$ (Table 1) have evolved towards minimal compact genomes and can bypass the need for the protein or any other chaperones in their riboregulation, probably because of evolutionary constraints linked to their ecological niches.

\section{Concluding remarks and future directions}

It is possible that additional chaperones could substitute for Hfq in bacteria lacking this protein. Database searches in $S$. aureus genomes for genes encoding Sm-like domains (which are structural signatures for RNA chaperones) reveal no other candidates than $h f q$. However, Gaballa and colleagues have recently reported that, in B. subtilis, an operon expresses three small basic proteins postulated to function as RNA chaperones (FbpA-FbpC) needed for the FsrA sRNA to regulate $s d h C$ expression [39]. These proteins are candidate substitutes for Hfq; a further search for proteins other than Hfq able to facilitate sRNA-mRNA interactions could lead to interesting findings. The requirement or dispensability of protein chaperones for a specific sRNA might depend on the pairings involved with each target mRNA and on the conformations of the interacting RNAs.

Based on an extensive set of experimental data collected from various bacterial species, we propose that a link exists between the need for Hfq in sRNA-mRNA interactions and (i) the overall GC content of bacterial genomes, (ii) the free energy for sRNA-mRNA pairing interactions, (iii) genome size and (iv) structural variations among Hfq proteins. Although none of these parameters, individually, can be used to make detailed predictions on the involvement of Hfq for a specific case, we suggest that they should be considered in furthering our understanding of the variable implication of $\mathrm{Hfq}$ in sRNA-mRNA regulation.

\section{Acknowledgments}

$\Delta h f q$ strains were gracefully given by Dr P. Bouloc (CNRS, Orsay). We thank Dr M. Hallier from our laboratory for his insightful comments. Our work is supported by a grant from the Région Bretagne CPER "Agent pathogène et risques et maladies infectieuses" and by an ANR606-MIME016-01 Microbiologie, Immunologie et Maladies émergentes grant to B.F. A.J. was supported by a fellowship from the Région Bretagne and by grant ANR606-MIME-016-01.

\section{References}

1 Repoila, F. and Darfeuille, F. (2009) Small regulatory non-coding RNAs in bacteria: physiology and mechanistic aspects. Biol. Cell 101, 117-131

2 Romby, P. et al. (2006) The role of RNAs in the regulation of virulencegene expression. Curr. Opin. Microbiol. 9, 229-236

3 Franze de Fernandez, M.T. et al. (1968) Factor fraction required for the synthesis of bacteriophage Q $\beta$-RNA. Nature 219, 588-590

4 Sun, X. et al. (2002) Predicted structure and phyletic distribution of the RNA-binding protein Hfq. Nucleic Acids Res. 30, 3662-3671

5 Schumacher, M.A. et al. (2002) Structures of the pleiotropic translational regulator Hfq and an Hfq-RNA complex: a bacterial Sm-like protein. EMBO J. 21, 3546-3556

6 Brescia, C.C. et al. (2003) Identification of the Hfq-binding site on DsrA RNA: Hfq binds without altering DsrA secondary structure. RNA 9, 33-43

7 Kajitani, M. et al. (1994) Regulation of the Escherichia coli hfq gene encoding the host factor for phage Q $\beta$. J. Bacteriol. 176, 531-534

8 Folichon, M. et al. (2003) The poly(A) binding protein Hfa protects RNA from RNase E and exoribonucleolytic degradation. Nucleic Acids Res. 31, 7302-7310

9 Tsui, H.C. et al. (1994) Characterization of broadly pleiotropic phenotypes caused by an $h f q$ insertion mutation in Escherichia coli K-12. Mol. Microbiol. 13, 35-49

10 Bohn, C. et al. (2007) No detectable effect of RNA-binding protein Hfa absence in Staphylococcus aureus. BMC Microbiol 7, 10

11 Boisset, S. et al. (2007) Staphylococcus aureus RNAIII coordinately represses the synthesis of virulence factors and the transcription regulator Rot by an antisense mechanism. Genes Dev. 21, 1353-1366

12 Majdalani, N. et al. (2005) Bacterial small RNA regulators. Crit. Rev. Biochem. Mol. Biol. 40, 93-113

13 Darfeuille, F. et al. (2007) An antisense RNA inhibits translation by competing with standby ribosomes. Mol. Cell 26, 381-392

14 Castillo-Keller, M. et al. (2006) Novel mechanism of Escherichia coli porin regulation. J. Bacteriol. 188, 576-586

15 Kawano, M. et al. (2007) An antisense RNA controls synthesis of an SOSinduced toxin evolved from an antitoxin. Mol. Microbiol. 64, 738-754

16 Arluison, V. et al. (2007) Spectroscopic observation of RNA chaperone activities of $\mathrm{Hfq}$ in post-transcriptional regulation by a small noncoding RNA. Nucleic Acids Res. 35, 999-1006

17 Sittka, A. et al. (2008) Deep sequencing analysis of small noncoding RNA and mRNA targets of the global post-transcriptional regulator. Hfq. PLoS Genet. 4, e1000163

18 Viegas, S.C. et al. (2007) Characterization of the role of ribonucleases in Salmonella small RNA decay. Nucleic Acids Res. 35, 7651-7664

19 Sonnleitner, E. et al. (2008) Detection of small RNAs in Pseudomonas aeruginosa by RNomics and structure-based bioinformatic tools. Microbiology 154, 3175-3187

20 Urban, J.H. and Vogel, J. (2007) Translational control and target recognition by Escherichia coli small RNAs in vivo. Nucleic Acids Res. 35, 1018-1037

21 Morita, T. et al. (2005) RNase E-based ribonucleoprotein complexes: mechanical basis of mRNA destabilization mediated by bacterial noncoding RNAs. Genes Dev. 19, 2176-2186

22 Moll, I. et al. (2003) Coincident Hfq binding and RNase E cleavage sites on mRNA and small regulatory RNAs. RNA 9, 1308-1314

23 Christiansen, J.K. et al. (2006) Identification of small Hfq-binding RNAs in Listeria monocytogenes. RNA 12, 1383-1396

24 Sonnleitner, E. et al. (2006) Hfq-dependent alterations of the transcriptome profile and effects on quorum sensing in Pseudomonas aeruginosa. Mol. Microbiol. 59, 1542-1558

25 Morita, T. et al. (2006) Translational repression is sufficient for gene silencing by bacterial small noncoding RNAs in the absence of mRNA destruction. Proc. Natl. Acad. Sci. U. S. A. 103, 4858-4863 
26 Lenz, D.H. et al. (2004) The small RNA chaperone Hfq and multiple small RNAs control quorum sensing in Vibrio harveyi and Vibrio cholerae. Cell 118, 69-82

27 Hammer, B.K. and Bassler, B.L. (2007) Regulatory small RNAs circumvent the conventional quorum sensing pathway in pandemic Vibrio cholerae. Proc. Natl. Acad. Sci. U. S. A. 104, 11145-11149

28 Song, T. et al. (2008) A new Vibrio cholerae sRNA modulates colonization and affects release of outer membrane vesicles. Mol. Microbiol. 70, 100-111

29 Mandin, P. et al. (2007) Identification of new noncoding RNAs in Listeria monocytogenes and prediction of mRNA targets. Nucleic Acids Res. 35, 962-974

30 Nielsen, J.S. et al. (2008) Identification of a $\sigma^{\mathrm{B}}$-dependent small noncoding RNA in Listeria monocytogenes. J. Bacteriol. 190, 6264-6270

31 Novick, R.P. et al. (1993) Synthesis of staphylococcal virulence factors is controlled by a regulatory RNA molecule. EMBO J. 12, 3967-3975

32 Pichon, C. and Felden, B. (2005) Small RNA genes expressed from Staphylococcus aureus genomic and pathogenicity islands with specific expression among pathogenic strains. Proc. Natl. Acad. Sci. U. S. A. $102,14249-14254$

33 Huntzinger, E. et al. (2005) Staphylococcus aureus RNAIII and the endoribonuclease III coordinately regulate spa gene expression. EMBO J. 24, 824-835

34 Geisinger, E. et al. (2006) Inhibition of rot translation by RNAIII, a key feature of agr function. Mol. Microbiol. 61, 1038-1048

35 Silvaggi, J.M. et al. (2005) Small untranslated RNA antitoxin in Bacillus subtilis. J. Bacteriol. 187, 6641-6650

36 Silvaggi, J.M. et al. (2006) Genes for small, noncoding RNAs under sporulation control in Bacillus subtilis. J. Bacteriol. 188, 532-541

37 Heidrich, N. et al. (2006) The small untranslated RNA SR1 from the Bacillus subtilis genome is involved in the regulation of arginine catabolism. Mol. Microbiol. 62, 520-536

38 Heidrich, N. et al. (2007) In vitro analysis of the interaction between the small RNA SR1 and its primary target $a h r C$ mRNA. Nucleic Acids Res. $35,4331-4346$

39 Gaballa, A. et al. (2008) The Bacillus subtilis iron-sparing response is mediated by a Fur-regulated small RNA and three small, basic proteins. Proc. Natl. Acad. Sci. U. S. A. 105, 11927-11932

40 Duhring, U. et al. (2006) An internal antisense RNA regulates expression of the photosynthesis gene isiA. Proc. Natl. Acad. Sci. U. S. A. $103,7054-7058$

41 Dienst, D. et al. (2008) The cyanobacterial homologue of the RNA chaperone Hfq is essential for motility of Synechocystis sp. PCC 6803. Microbiology 154, 3134-3143

42 Geissmann, T.A. and Touati, D. (2004) Hfa, a new chaperoning role: binding to messenger RNA determines access for small RNA regulator. EMBO J. 23, 396-405

43 Kawamoto, H. et al. (2006) Base-pairing requirement for RNA silencing by a bacterial small RNA and acceleration of duplex formation by Hfq. Mol. Microbiol. 61, 1013-1022

44 Wagner, E.G. and Flardh, K. (2002) Antisense RNAs everywhere? Trends Genet. 18, 223-226

45 Muckstein, U. et al. (2006) Thermodynamics of RNA-RNA binding. Bioinformatics 22, 1177-1182

46 Vecerek, B. et al. (2008) The C-terminal domain of Escherichia coli Hfq is required for regulation. Nucleic Acids Res. 36, 133-143
47 Nielsen, J.S. et al. (2007) An Hfq-like protein in archaea: crystal structure and functional characterization of the Sm protein from Methanococcus jannaschii. RNA 13, 2213-2223

48 Brennan, R.G. and Link, T.M. (2007) Hfq structure, function and ligand binding. Curr. Opin. Microbiol. 10, 125-133

49 Axmann, I.M. et al. (2005) Identification of cyanobacterial non-coding RNAs by comparative genome analysis. Genome Biol. 6, R73

50 Steglich, C. et al. (2008) The challenge of regulation in a minimal photoautotroph: non-coding RNAs in Prochlorococcus. PLoS Genet. 4, e1000173

51 Ostberg, Y. et al. (2004) The etiological agent of Lyme disease, Borrelia burgdorferi, appears to contain only a few small RNA molecules. $J$. Bacteriol. 186, 8472-8477

52 Kreikemeyer, B. et al. (2001) Group A streptococcal growth phaseassociated virulence factor regulation by a novel operon (Fas) with homologies to two-component-type regulators requires a small RNA molecule. Mol. Microbiol. 39, 392-406

53 Roberts, S.A. and Scott, J.R. (2007) RivR and the small RNA RivX: the missing links between the CovR regulatory cascade and the Mga regulon. Mol. Microbiol. 66, 1506-1522

54 Mangold, M. et al. (2004) Synthesis of group A streptococcal virulence factors is controlled by a regulatory RNA molecule. Mol. Microbiol. 53, $1515-1527$

55 Sorger-Domenigg, T. et al. (2007) Distinct and overlapping binding sites of Pseudomonas aeruginosa Hfq and RsmA proteins on the noncoding RNA RsmY. Biochem. Biophys. Res. Commun. 352, 769-773

56 Zhang, A. et al. (2003) Global analysis of small RNA and mRNA targets of Hfq. Mol. Microbiol. 50, 1111-1124

57 Landt, S.G. et al. (2008) Small non-coding RNAs in Caulobacter crescentus. Mol. Microbiol. 68, 600-614

58 Metruccio, M.M. et al. (2009) The Hfq-dependent small noncoding RNA NrrF directly mediates Fur-dependent positive regulation of succinate dehydrogenase in Neisseria meningitidis. J. Bacteriol. 191, 1330-1342

59 Pfeiffer, V. et al. (2007) A small non-coding RNA of the invasion gene island (SPI-1) represses outer membrane protein synthesis from the Salmonella core genome. Mol. Microbiol. 66, 1174-1191

60 Sharma, C.M. et al. (2007) A small RNA regulates multiple ABC transporter mRNAs by targeting C/A-rich elements inside and upstream of ribosome-binding sites. Genes Dev. 21, 2804-2817

61 Swiercz, J.P. et al. (2008) Small non-coding RNAs in Streptomyces coelicolor. Nucleic Acids Res. 36, 7240-7251

62 Grieshaber, N.A. et al. (2006) A small RNA inhibits translation of the histone-like protein Hc1 in Chlamydia trachomatis. Mol. Microbiol. 59, $541-550$

63 Gruber, A.R. et al. (2008) The Vienna RNA websuite. Nucleic Acids Res. 36 , W70-74

64 Pulvermacher, S.C. et al. (2009) Role of the Escherichia coli Hfa protein in GcvB regulation of oppA and $d p p A$ mRNAs. Microbiology 155, 115123

65 Altuvia, S. et al. (1998) The Escherichia coli OxyS regulatory RNA represses fhlA translation by blocking ribosome binding. EMBO J. 17, $6069-6075$

66 Moller, T. et al. (2002) Spot 42 RNA mediates discoordinate expression of the E. coli galactose operon. Genes Dev. 16, 1696-1706

67 Udekwu, K.I. et al. (2005) Hfq-dependent regulation of OmpA synthesis is mediated by an antisense RNA. Genes Dev. 19, 2355-2366 